\section{P174 Classroom Nutrition Education Combined With USDA's Fruit and Vegetable Snack Program Improves Children's Fruit and Vegetable Intake}

Abby Gold, PhD, MPH, RD, abby.gold@ndsu.edu, North Dakota State University, Department 7270, PO Box 6050, Fargo, ND 58108; M. Larson, PhD, MPH, RD, CHES, CDE; J. Simpson, PhD, Helen DeVos Children's Hospital; M. Strang, MS, RD, North Dakota State University Extension Service

Objective: This study tests the effectiveness of a schoolbased intervention and curriculum, Go Wild with Fruits and Veggies! (GWWFV), on fruit and vegetable (FV) intake. GWWFV curriculum is a 7-lesson curriculum created for 3rd - 5th graders, including taste-testing, classroom activities, take-home challenges, and parent newsletters.

Design, Setting and Participants: A randomized control trial tested self-reported FV intake in children. Volunteer schools were randomized into control (12 schools, $\mathrm{n}=325$ children), or intervention (14 schools, $\mathrm{n}=337$ children). Extension educators administered the curriculum within intervention schools.

Outcome Measures and Analysis: Children completed 2 validated pre/post-intervention surveys: a food behavior checklist and FV intake survey. Control group took the surveys over the same pre/post time-period. Access to the federal Fruit and Vegetable Snack Program (FVSP) and taste-testing within GWWFV were also measured. Baseline nutritional behaviors in children according to enrollment in USDA snack program were analyzed using descriptive statistics. Behavior change according to enrollment in GWWFV and taste test intervention were analyzed using relative risk and t-tests.

Results: Students with access to the FVSP consumed significantly more fruits $(\mathrm{p}<.01)$ and vegetables $(\mathrm{p}<.0001)$. Students who participated in the FVSP and GWWFV with taste-testing consumed more fruits and vegetables $(\mathrm{p}<.05)$ compared to students who participated in the FVSP and GWWFV without taste-testing, and the control group.

Conclusions and Implications: The FVSP and GWWFV with taste-testing demonstrated a greater positive influence on FV intake. Environmental and educational strategies form a comprehensive approach to improving children's diets. Offering hands-on nutrition education curricula alongside the FVSP may result in greater FV intake in children.

Funding: None.

\section{P175 Relationship Between Mothers' Concerns for Lunch Boxes, Children's Lunch Boxes Contents and Children's Preferences in Japan}

Tomoko Osera,PhD,RD, tomokocup12@yahoo.co.jp, Kobe Women's University, Hygiene and Preventive Medicine, Graduate School of Life Science, 2-1 Higashisuma-Aoyama, Suma Kobe, 654-8585, Japan; S. Tsutie, MS, RD; K. Nishikawa, BS; Y. Segawa, MS, RD; M. Kobayashi, MS; Takakuradai Kindergarten;

N. Kurihara, PhD, Kobe Women's University
Objective: Japanese kindergarten children usually bring a lunch box (LB), which their mothers usually make. The contents may be influenced by the mothers' food concern. We investigated the relationship between mothers' concern, children's LB contents and children's preferences. Design, Setting and Participants: LBs of 210 children were digitally photographed 4 times over the course of a school year at a private kindergarten in Japan. The amounts of rice, main dishes, vegetables and fruits were then estimated by measuring the area in photograph. At the end of the investigation, a questionnaire, including questions about mother's concern and children's preferences, was given to the mothers.

Outcome Measures and Analysis: Data were analyzed together by Fisher's exact test and Mann-Whitney U test. Results: Percentages of estimated amounts of rice, main dishes, vegetables and fruits in LBs were $37.9 \pm 9.2 \%, 37.3$ $\pm 10.0 \%, 11.9 \pm 7.8 \%$ and $8.9 \pm 7.9 \%$, respectively. The estimated vegetable amount was less than the Japanese dietary reference intake. Mothers' concerns for children's LBs were significantly related to vegetable amounts in the LBs $(\mathrm{p}<0.01)$. When vegetable amounts were estimated to be over $11 \%$, (1) the number of foods which children disliked was less, (2) the mothers reported a higher rate of mindfulness toward vegetables, and (3) a lower rate of frozen food usage and thought they made balanced LBs, compared with when below $11 \%(\mathrm{p}<0.01$, each). Thus, vegetable amounts in children's LB estimated by photograph may predict mothers' food concerns and children's balanced/unbalanced diets.

Conclusions and Implications: Vegetable amounts in LB may be related to the mothers' concern for vegetables and children's preferences.

Funding: None.

\section{P176 Food and Drink Free Lists Document Home Environments and Create Content for Messages to Parents of Middle-School Children}

Erin Green, RD, emg232@cornell.edu, Cornell University, Division of Nutritional Sciences, 408 Savage Hall, Ithaca, NY 14853; T. Hill, MPH; A. Gaines, PhD; J. Dollahite, PhD, RD

Objective: Recent formative research among parents of middle-school children suggests that getting children to try new vegetables and fruits, eat a variety of vegetables, and choose water over sugar-sweetened beverages is particularly challenging. To provide parents with relevant information and feasible strategies to support children in making healthful choices, a better understanding of current food and drink exposure is needed. This study explored reported fruit, vegetable, and drink variety at home.

Design, Setting and Participants: In-depth interviews were conducted with 17 parents $(1 \mathrm{M} ; 16 \mathrm{~F})$ of middleschool children and 21 middle-school children $(11 \mathrm{M} ; 10$ F). A modified free listing technique was used to record current fruits, vegetables, and drinks consumed or available to respondents. 